\title{
Uso de amitriptilina como coadyuvante en el manejo de la enfermedad por reflujo gastroesofágico
}

\section{Amitriptyline as an adjunct in the management of gastroesophageal reflux disease}

DOI: https://doi.org/10.36104/amc.2021.2246

\author{
Señores editores “Acta Médica Colombiana” \\ Cordial saludo.
}

Después de leer con detenimiento el artículo titulado "Impacto de la optimización del tratamiento en pacientes con Enfermedad por Reflujo Gastroesofágico que no responden a esomeprazol" (1), de forma respetuosa hago las siguientes observaciones.

En el estudio mencionado es notorio que la mayoría de pacientes estaban en el grupo de edad entre 60-74 años $(43.2 \%)$ o sea corresponden a adultos mayores. La formulación de medicamentos en este grupo de edad entraña particularidades relacionadas con los cambios fisiológicos de la edad avanzada, la polifarmacia, la polipatología, además de circunstancias cognitivo emocionales y sociales.

Los criterios de Beers (2), constituyen una herramienta ampliamente usada en la práctica geriátrica en donde se define por consenso de un grupo de expertos los medicamentos potencialmente inapropiados para ser usados por adultos mayores y entre los cuales la amitriptilina ocupa un lugar destacado.

La amitriptilina, además de bloquear la recaptación de serotonina y noradrenalina, tiene alta afinidad por los receptores de histamina $\mathrm{H} 1$ y muscarínicos $\mathrm{M} 1$ y así como otros antidepresivos tricíclicos, se usa cada vez menos, sobre todo desde la aparición de los inhibidores de recaptación de serotonina (3).

$\mathrm{Su}$ perfil desfavorable de reacciones adversas medicamentosas, siendo más relevantes en adultos mayores, particularmente anticolinérgicos (boca seca, visión borrosa, estreñimiento), alfa adrenérgicos (efectos cardiacos, prolongación del intervalo QT , arritmias e hipotensión ortostática), sedantes (aumentando el riesgo de caídas, fracturas y delirium), además de disminución del umbral convulsivo, disfunción sexual, diaforesis y temblor. Estos efectos que son dependientes de la dosis, pero en ancianos se presentan desde dosis bajas; de hecho la interrupción del tratamiento debido a efectos secundarios ocurrió con más frecuencia con tricíclicos de dosis baja (4).

Además la eficacia de los antidepresivos tricíclicos como neuromoduladores viscerales, en particular amitriptilina, ha sido altamente cuestionada (5), de hecho en las "Directrices globales de la Organización Mundial de Gastroenterología", sobre el manejo de la enfermedad por reflujo gastroesofágico en su versión del 2017 (6) y en las "guías prácticas basadas en la evidencia para enfermedad por reflujo gastroesofágico de la Sociedad Japonesa de Gastroenterología del año 2015 (7), no la proponen dentro del arsenal terapéutico.

En conclusión, los efectos secundarios de los antidepresivos tricíclicos generalmente los hacen menos tolerables en comparación con los inhibidores selectivos de la recaptación de serotonina (ISRS) y otros antidepresivos más nuevos (8), por tanto en el caso de requerir un antipresivo como modulador visceral, la elección se inclinaría por estos últimos grupos.

Dr. Alexánder Morales-Eraso

Internista, Geriatra. MSc Epidemiología. Doctorando en dirección de proyectos Docente Investigador grupo GIISE Universidad Cooperativa de Colombia, campus Pasto Hospital Universitario Departamental de Nariño

Correspondencia: Dr. Alexánder Morales-Eraso E-mail: alexandermoraleserazo@gmail.com

\section{Referencias}

1. Lozano-Martinez J, Otero-Regino W, Marulanda-Fernadez H. Impacto de la optimización del tratamiento en pacientes con EnfeCordialrmedad por Reflujo Gastroesofágico que no responden a Esomeprazol. Acta Med Colomb Vol. 46 Núm. 2 (2021).

2. American Geriatrics Society 2019 Actualizado AGS Beers Criteria ${ }^{\circledR}$ para el uso de medicamentos potencialmente inapropiados en adultos mayores. Am Geriatr Soc 2019; 67 (4): 674.

3. Hirsch M, Birnbaum R. Fármacos tricíclicos y tetracíclicos: farmacología administración y efectos secundarios. UpToDate. Acceso en línea: 06/07/2021.

4. Furukawa TA, McGuire H, Barbui C. Metaanálisis de los efectos y efectos secundarios de los antidepresivos tricíclicos de dosis baja en la depresión: revisión sistemática. BMJ 2002; 325 (7371): 991.

5. Weijenborg PW, de Schepper HS, Smout AJPM, Bredenoord AJ. Effects of Antidepressants in Patients With Functional Esophageal Disorders or Gastroesophageal Reflux Disease: A Systematic Review. Clin Gastroenterol Hepatol 2015; 13(2):251-259.e1

6. Hunt R, Armstrong D, Katelaris P. Perspectiva global de ERGE sobre la enfermedad por reflujo gastroesofágico Directrices globales de la Organización Mundial de Gastroenterología. Journal of Clinical Gastroenterology: julio de 2017 - Volumen 51 - Número 6 - p 467-478.

7. Iwakiri K, Kinoshita Y, Habu Y, et al. Evidence-based clinical practice guidelines for gastroesophageal reflux disease 2015. J Gastroenterol 2016; 51(8): 751-767.

8. Anderson IM, Ferrier IN, Baldwin RC, Cowen PJ, Howard L. Directrices basadas en la evidencia para el tratamiento de los trastornos depresivos con antidepresivos: una revisión de las directrices de la Asociación Británica de Psicofarmacología de 2000. J Psychopharmacol 2008; 22 (4): 343. Epub 2008 15 de abril. 


\section{RESPUESTA}

\section{Pirosis no es lo mismo que enfermedad por reflujo gastroesofágico (ERGE)}

\section{Heartburn is not the same as gastroesophageal reflux disease (GERD)}

Apreciado Señor Editor.

Agradecemos el interés del Dr. Alexánder Morales-Eraso por leer nuestro artículo "Impacto de la optimización del tratamiento en pacientes con enfermedad por reflujo gastroesofágico que no responden a esomeprazol" publicado recientemente en le revista (1).

El objetivo de nuestro trabajo era determinar si la optimización del tratamiento en los pacientes con ERGE, podía controlar los síntomas. La optimización consistió en verificar y corregir la dosis del inhibidor de bomba de protones (IBP), hacer las recomendaciones sobre "medidas generales" con eficacia demostrada en el tratamiento de la ERGE (disminuir de peso si el índice de masa corporal es $\geq 25$, dejar de fumar, controlar el estrés) $(2,3)$ y prescribir una segunda dosis del IBP antes de la cena. Si los pacientes continuaban con pirosis (ardor retroesternal), se les adicionaba una dosis baja de un "neuromodulador visceral". Para el estudio, elegimos amitriptilina en dosis de 12.5 a $25 \mathrm{mg}$ al acostarse, porque ha sido previamente utilizado en trastornos funcionales gastrointestinales $(4,5)$. La decisión de utilizar el neuromodulador se basa en la demostración previa, de que $75-90 \%$ de los pacientes con ERGE, que continúan con pirosis a pesar de dos dosis de IBP, se debe a coexistencia de un trastorno funcional (hoy llamados desórdenes de la interacción del eje intestino-cerebro, tales como pirosis funcional o hipersensibilidad al reflujo (6-9). En esas entidades existe hiperalgesia o "alodinia" en el esófago, que explica la pirosis $(4,8,9)$. Esas entidades producen pirosis, similar a otras patologías diferentes a la ERGE $(4,6,8)$ y por lo tanto pirosis no es lo mismo que ERGE $(4,6,8,10)$. En nuestro trabajo, el neuromodulador se adicionó cuando la pirosis era refractaria, es decir, que persistía a pesar de dos dosis de IBP durante 8-12 semanas, correctamente formulados. Como se puede comprender, el antidepresivo a dosis bajas o "neuromodulador visceral", que es el nombre utilizado cuando se utilizan dosis inferiores a las antidepresivas (4, $6,12)$, no se ha utilizado para tratar la ERGE sino para la "pirosis refractaria". La piedra angular del tratamiento de la ERGE es la inhibición de la secreción de ácido con IBP y esto coinciden todas las guías internaciones y expertos ( 3 , $4,9,12,13)$, además de las guías que menciona el doctor Morales. En ninguna guía se recomiendan los neuromoduladores viscerales o antidepresivos para el manejo de la ERGE, como tampoco lo hacemos nosotros. El objetivo del estudio era determinar la eficacia de un abordaje secuencial en pacientes con síntomas de ERGE refractarios, incluyendo al final un neuromodulador (1). El abordaje de los pacientes con pirosis refractaria y ERGE, es mucho más amplio e incluye adicionar procinéticos, tomar biopsias del esófago para descartar esofagitis eosinofílica, $\mathrm{pH}$ metría esofágica con impedancia $(4,8,9,11,12)$ y más recientemente colesevelam un adsorbente de los ácidos biliares a nivel gástrico (13).

Con respecto al uso de antidepresivos en el paciente adulto mayor ( $\geq 65$ años), estamos de acuerdo con el doctor Morales, pero en nuestro estudio no hemos utilizado estos medicamentos a dosis antidepresivas. La eficacia de los "neuromoduladores viscerales" en pirosis refractaria o hipersensibilidad al reflujo, no se ha demostrado para todos los antidepresivos. Existen muchas opciones propuestas tales como antidepresivos tricíclicos (imipramina, amitriptilina), inhibidores selectivos de recaptación de serotonina (citalopram, fluoxetina, sertralina), inhibidores de recaptación de serotonina y norepinefrina (venlafaxina, duloxetine) $(8$, $10,12)$. Ninguno de esos medicamentos ha sido aprobado para el manejo de los trastornos funcionales esofágicos (4, $5,8)$. Sin embargo, dada la experiencia previa con ellos en otras patologías, previo consentimiento informado, pueden utilizarse "off label". Elegimos la amitriptilina por su mayor efecto analgésico, bajo costo y perfil de seguridad a dosis bajas $(5,11,13)$. Estamos de acuerdo que tienen potencial pro arrítmico por su efecto sobre los canales rápidos de sodio, lo cual amerita un electrocardiograma pretratamiento para identificar factores de riesgo (prolongación del QT, bloqueo de rama izquierda, bloqueo bifascicular). Estos pacientes fueron excluidos de nuestro estudio (1). Los inhibidores de recaptación de serotonina son una opción y hay algunos estudios preliminares en hipersensibilidad al reflujo (15). Sin embargo, en un metaanálisis reciente en pacientes con dispepsia funcional, los inhibidores selectivos de la recaptación de serotonina (ISRS) no mostraron un beneficio sobre el placebo $(\mathrm{RR}=1.00, \mathrm{IC} 95 \% 0.86-1.17, \mathrm{I} 2=0 \%$, $\mathrm{p}=0.82$ ) (15). En nuestra investigación se utilizaron dosis de $\leq 25 \mathrm{mg}$ de amitriptilina en pacientes con síntomas de ERGE refractarios, que es inferior a la dosis sugerida para otros trastornos funcionales $(25-100 \mathrm{mg} /$ día $)(4,5,10)$. Nuestro enfoque es un escenario hasta ahora no explorado en gastroenterología, pero sí sugerido por expertos $(6,8)$. Es una exploración en el manejo de esos pacientes en nuestro 
medio, teniendo en cuenta los altos costos de los estudios de fisiología esofágica.

Agradecemos al Dr. Morales su interés en leer nuestro trabajo y coincidimos en la importancia de tener en cuenta los efectos adversos cardiovasculares de la amitriptilina en los adultos mayores, por lo cual se utilizaron dosis bajas de acuerdo con lo recomendado $(4,5,15)$.

\section{Cordialmente}

\section{Dr. William Otero-Regino, FAGA FACP Dr. Hernando Marulanda-Fernández Universidad Nacional de Colombia Hospital Universitario Nacional de Colombia.}

Correspondencia: Dr. William Otero-Regino, Dr. Hernando Marulanda E-mail: wotero@cable.net.co,hmarulandafe@unal.edu.co

\section{Referencias}

1. Lozano JE, Otero W, Marulanda H. Impacto de la optimización del tratamiento en pacientes con enfermedad por reflujo gastroesofágico que no responden Acta Med Colomb 2021; 46. DOI: https://doi.org/10.36104/amc.2021.2041..

2. Castillo R, Otero W, Trespalacios AA. Impacto de las medidas generales en el tratamiento del reflujo gastroesofágico: una revisión basada en la evidencia. Rev Col Gastroenterol. 2015;30(4):431-6.

3. Katz PO, Gerson LB, Vela MF. Guidelines for the Diagnosis and Management of Gastroesophageal Reflux Disease. Am J Gastroenterol 2013; 108:308-28.

4. Aziz Q, Fass R, Gyawali CP, Miwa H, Pandolfino JE, Zerbib F. Esophageal disorders. Gastroenterology. 2016;150(6):1368-79.
5. Törnblom H, Drossman DA. Psychotropics, Antidepressants, and Visceral Analgesics in Functional Gastrointestinal Disorders. Curr Gastroenterol Rep. 2018;20(12):58. doi: 10.1007/s11894-018-0664-3.

6. Abdallah J, George N, Yamasaki T, Ganocy S, Fass R. Most Patients With Gastroesophageal Reflux Disease Who Failed Proton Pump Inhibitor Therapy Also Have Functional Esophageal Disorders. Clin Gastroenterol Hepatol. 2019;17(6):1073-1080.

7. Roman S, Keefer L, Imam H, Korrapati P, Mogni B, Eident K, et al. Majority of symptoms in esophageal reflux PPI non-responders are not related to reflux. Neurogastroenterol Motil. 2015;27(11):1667-74.

8. Fass OZ, Fass R. Overlap Between GERD and Functional Esophageal Disorders a Pivotal Mechanism for Treatment Failure. Curr Treat Options Gastroenterol. 2019;17(1):161-164.

9. Gyawali CP, Fass R. Management of Gastroesophageal Reflux Disease Gastroenterology 2018;154:302-318

10. Gabard S, Vijayvargiya S. Functional heartburn: An underrecognized cause of PPI-refractory symptoms. Clev Clin J Med 2019; 86 (12):799-806

11. Fass R, Zerbib F, Gyawali P. AGA Clinical Practice Update on Functional Heartburn: Expert Review. Gastroenterology 2020;158:2286-2293.

12. Talley NJ, Zand Irani M. Optimal management of severe symptomatic gastroesophageal reflux disease. J Intern Med. 2021;289(2):162-178.

13. Vaezi MF, Fass R, Vakil N, Reasner DS, Mittleman RS, Hall M, Shao JZ, Chen Y, Lane L, Gates AM, Currie MG. IW-3718 Reduces Heartburn Severity in Patients With Refractory Gastroesophageal Reflux Disease in a Randomized Trial. Gastroenterology. 2020;158(8):2093-2103. doi: 10.1053/j.gastro.2020.02.031

14. Viazis N, Keyoglou A, Kanellopoulos AK, et al. Selective serotonin reuptake inhibitors for the treatment of hypersensitive esophagus: a randomized, doubleblind, placebocontrolled study. Am J Gastroenterol 2012;107:1662-1667.

15. Lacy BE, Saito YA, Camilleri M, Bouras E, DiBaise JK, Herrick LM, Szarka LA, Tilkes K, Zinsmeister AR, Talley NJ. Effects of Antidepressants on Gastric Function in Patients with Functional Dyspepsia. Am J Gastroenterol. 2018;113(2):216-224. doi: 10.1038/ajg.2017.458. 